\title{
The role of dimensionality on the quenching of spin-orbit effects in the optics of gold nanostructures
}

\author{
Alberto Castro, ${ }^{1, a)}$ Miguel A. L. Marques, ${ }^{2}$ Aldo H. Romero, ${ }^{3}$ Micael J. T. Oliveira, ${ }^{4}$ and \\ Angel Rubio ${ }^{5}$ \\ ${ }_{1}^{1}$ Institut für Theoretische Physik and European Theoretical Spectroscopy Facility, Freie Universität Berlin, \\ Arnimallee 14, D-14195 Berlin, Germany \\ ${ }^{2}$ Laboratoire de Physique de la Matière Condensée et Nanostructures, Université Lyon 1, \\ CNRS, UMR 5586, Domaine scientifique de la Doua, F-69622 Villeurbanne Cedex, France \\ ${ }^{3}$ CINVESTAV Querétaro, Libramiento Norponiente No. 2000, 76230 Querétaro, Mexico \\ ${ }^{4}$ Centre of Computational Physics, University of Coimbra, Rua Larga, 3004-516 Coimbra, Portugal \\ ${ }^{5}$ Nano-Bio Spectroscopy Group and European Theoretical Spectroscopy Facility, \\ Departamento de Fisica de Materiales, Facultad de Químicas, Centro Mixto CSIC-UPV/EHU, \\ and DIPC, Universidad del País Vasco, Edificio Korta, 20018 San Sebastián, Spain
}

(Received 22 July 2008; accepted 26 August 2008; published online 13 October 2008)

\begin{abstract}
By first-principles time-dependent density-functional calculations, we show the relevance of relativistic effects to shape the photoabsorption cross section of small gold clusters $\left(\mathrm{Au}_{n}, n \leqslant 8\right.$, and $n=20)$ and small nanowires $(n \leqslant 7)$. The relativistic effects not only dictate the stabilization of planar geometries (as it has already been shown by treating the core electrons relativistically): The spin-orbit coupling also has a strong impact in the absorption spectra (resonances and oscillator strengths). This is especially true for nanowires, where the effect of spin orbit is large and not substantially reduced with the chain length, in contrast to more compact gold clusters where this spin-orbit effect tends to be quenched. These results have far reaching consequences in fields such as electronic transport, where gold nanowires are often used, but where spin-orbit effects are generally disregarded. (C) 2008 American Institute of Physics. [DOI: 10.1063/1.2990745]
\end{abstract}

The road towards well-defined self-assembled nanostructured devices with tailored properties has renewed the interest in the electronic and structural properties of isolated and supported clusters. ${ }^{1}$ Nanoparticles are of interest due to their special catalytic properties, ${ }^{2}$ and due to their applicability in optical devices: ${ }^{3}$ as the optical response for nanometer structures depends crucially on the particle size due to quantum effects, their absorbing and emitting spectrum can be tuned by changing its size or shape. The research on (and possible application of) small size- and shape-selected coinage metal nanoparticles depends on the feasibility of their selective preparation. Fortunately, experimental breakthroughs in the last decades have demonstrated this possibility, e.g., for gold-which is the topic of this work-a synthesis method that allows for control of the average cluster size and deposits the gold nanostructures on $\mathrm{MgO}$ was reported in Ref. 4; previously, the large size dependence of the catalytic properties of gold clusters supported on magnesia was reported in Ref. 5. In a different example, functionalized $\mathrm{Au}_{55}$ clusters were generated using a "bottom-up" fabrication method based on the self-assembly of the clusters on organic template patterns. ${ }^{6}$

Gold is ubiquitous in surface science due to its inertness. However, the reduction in dimensionality revealed new and exciting phenomena and possibilities not at all present before: among others, enhanced reactivity, ${ }^{2}$ control of shape and size by external electric fields, ${ }^{7}$ new optical devices, ${ }^{8}$

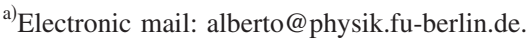

and applications as a basic ingredient for metamaterials or as an essential component in adaptive-shape femtosecond laser pulse generators.

Theoretically, the size dependence of the electronic, magnetic and chemical properties of 3D metallic nanoparticles is by now well understood. ${ }^{9}$ Still, the interpretation of the noble metal clusters absorption spectra is quite complex. Anionic silver clusters exhibit the expected redshift as the cluster size is decreased, ${ }^{10}$ whereas the cations are blueshifted. ${ }^{11}$ This blueshift has also been found in the optical response of large (2-4 nm diameter) gold nanoparticles supported in alumina, ${ }^{12}$ and can be traced back to the action of the $d$ electrons that form a (size-dependent) polarizable background that strongly screens the valence electron interactions. ${ }^{13}$

It is therefore clear that, even if the $d^{10} s^{1}$ electronic arrangement of all coinage metals may suggest their treatment as simple alkaline clusters, the $d$-shell interplay with the $s$ electron has an important contribution. However, this does not suffice to explain the singularity of gold nanostructures: relativistic effects were proven to be crucial for the color of gold and for understanding gold chemistry. ${ }^{14}$ The bonding in gold clusters is influenced by relativistic effects that tend to increase the degree of $s d$ hybridization in the molecular orbitals, modifying the geometries of gold clusters: planar structures are favored for small clusters up to a surprisingly large number of atoms (up to $n=7,11$, and 12 for cationic, neutral and anionic clusters, respectively. ${ }^{15-17}$ ) These numbers are much larger for gold than for copper or silver, and 


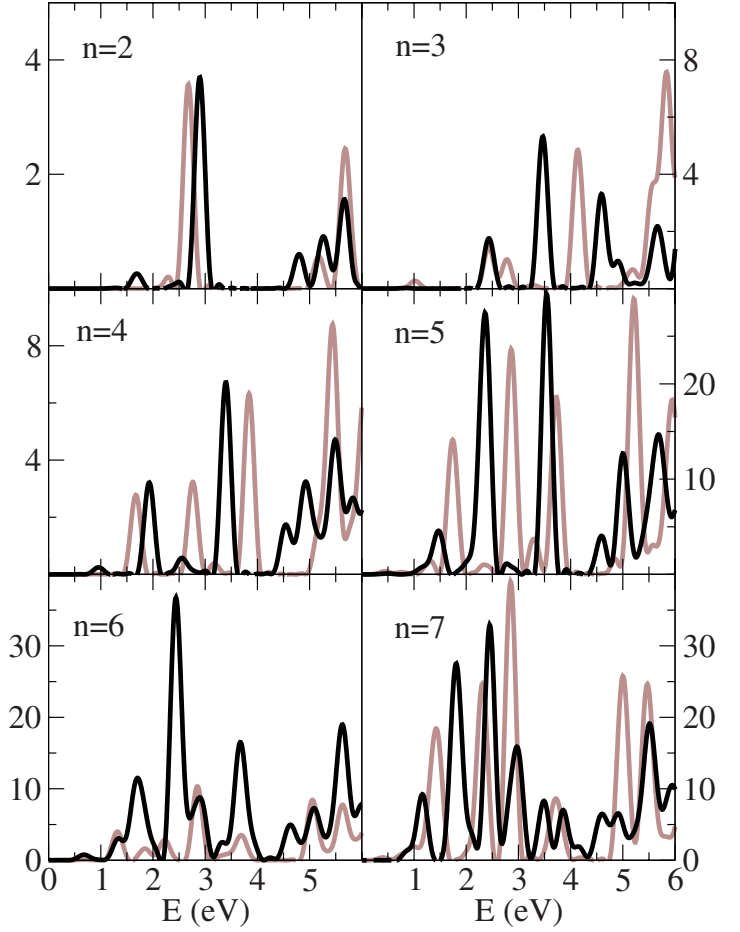

FIG. 1. (Color online) Absorption cross section (a.u.) of linear $\mathrm{Au}_{n}(n=2$ $\times 7$ ), obtained with (black line) and without (grey line) spin-orbit coupling.

the reason can be unambiguously assigned to relativity. ${ }^{18}$

Even though the necessity of including to some extent relativistic effects has always been stressed, the effect of spin-orbit coupling on the electronic excitations has been overseen. ${ }^{19}$ Usually, except for accurate (quasi)relativistic quantum chemistry calculations for the atom or the dimer, ${ }^{20}$ the account for relativity is restricted to using relativistic effective core potentials or pseudopotentials. ${ }^{21}$ Yet, the spinorbit splitting of the lowest-lying nonforbidden spectroscopic line of the gold atom is as large as $0.5 \mathrm{eV} .{ }^{22} \mathrm{Can}$ one expect that this splitting is quenched by increasing the size of the $\mathrm{Au}$ clusters? Or, that it is washed away as the density of states grows-and with it, the complexity of absorption spectra-so that the general shape and features of the spectra are largely unaffected by the presence of spin-orbit in the model? Does the magnitude of spin-orbit effects depend on the cluster dimensionality (wires, planar, or 3D structures)? These questions are the object of the present work. In particular, we will be looking at optical absorption spectra within time-dependent density-functional theory (TDDFT). ${ }^{23}$ This theory allows the calculation of excited-state energies and spectra involving neutral excitations, and has been quite successful describing the optical spectra of clusters. In particular, we used the explicit time-propagation technique, ${ }^{24}$ as implemented in the OCTOPUS code. ${ }^{25}$

The transition from planar to three dimensional structures in small gold clusters has been investigated previously (see, for example, Refs. 26 and 27). The aim of this work is to assess the spin-orbit effects as a function of dimensionality; For such purpose, we have obtained the geometries from Ref. 26 and reoptimized them through Car-Parrinello dynamics $^{28}$ with norm-conserving scalar-relativistic pseudopotentials. ${ }^{29}$ To obtain our 3D geometries we per-

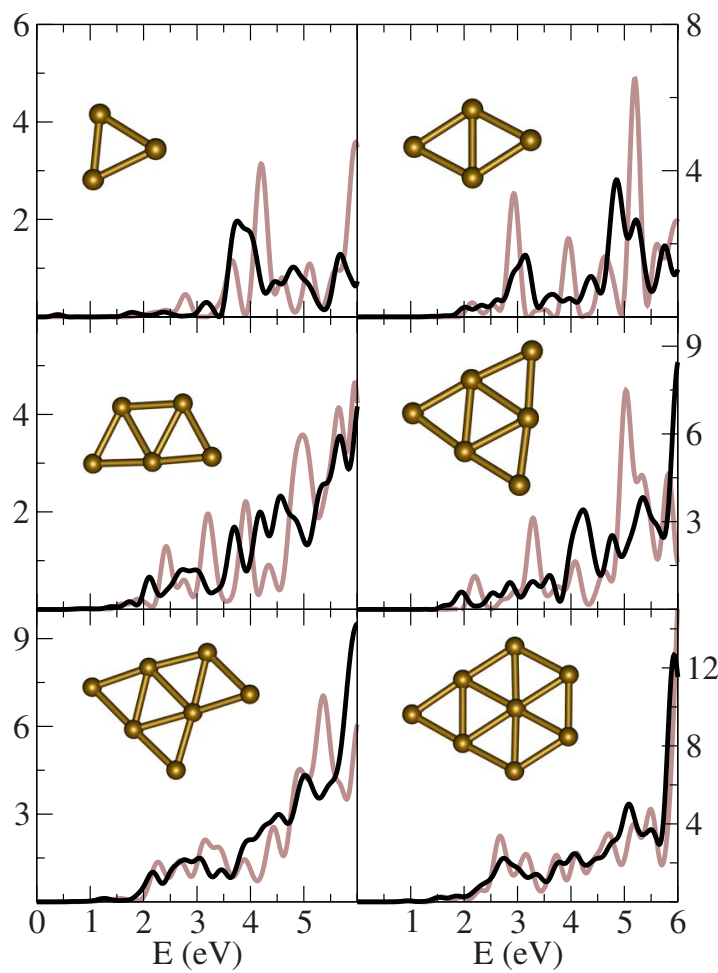

FIG. 2. (Color online) Absorption cross section (a.u.) of $2 \mathrm{D} \mathrm{Au}_{n}(n=3$ $\times 8$ ), obtained with (black line) and without (grey line) spin-orbit coupling.

formed a similar process as the one described in Ref. 31, where a classical potential in conjunction with genetic algorithms was used to obtain the initial starting points for the $\mathrm{CP}$ dynamics. At this stage the spin-orbit coupling was neglected, since (i) the structural modification introduced by this effect is small, and (ii) the aim of our work is to isolate spin-orbit effects in the electronic excitations (comparing the spectra with and without spin-orbit for the same geometry). It is important to treat exchange-correlation effects at the PBE-GGA level. ${ }^{30}$ Our ab initio calculations confirm that planar structures are the most stable for the cluster sizes studied here (except for the $\mathrm{Au}_{20}$ case). The obtained planar and 3D structures are shown in Figs. 1 and 2. In addition, to complete the study of the effect of dimensionality, we have performed computations for linear chains (wires) of gold atoms. For these 1D structures we chose to fix the bond length to $2.9 \AA$, which is the nearest-neighbor distance in bulk gold $^{32}$ (however, for the dimer we have used our computed equilibrium bond length, $2.55 \AA$, which compares well to the experimental value of $2.472 \AA$ ). ${ }^{33}$ Note that nanowire gold chains were predicted and generated experimentally in the 1990 s, and were the subject of extensive investigations. ${ }^{34,35}$ Low-dimensional gold structures are also widely used in the study of electronic transport through single molecules, often connecting the molecule to the leads and supported on surfaces. ${ }^{35}$

Regarding computational details, we represented the relevant functions on a real-space regular rectangular grid (the grid spacing was chosen to be 0.4 a.u.; the border of the simulation box was taken 8 a.u. away from the closest nucleus). The total propagation time was 750 a.u., and we used the (adiabatic) LDA to describe exchange-correlation 


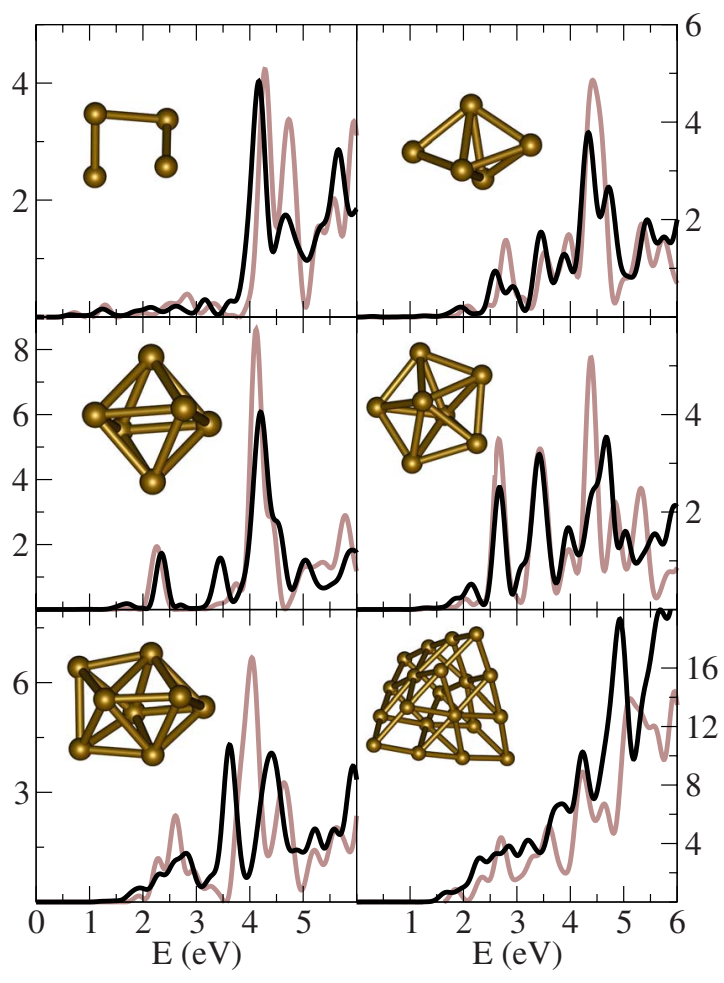

FIG. 3. (Color online) Absorption cross section (a.u.) of 3D $\mathrm{Au}_{n}(n=4$ $\times 7,20$ ), obtained with (black line) and without (grey line) spin-orbit coupling.

effects. ${ }^{36}$ We generated relativistic nonlocal norm-conserving pseudopotentials following Ref. 37, as implemented in the code APE. ${ }^{38}$ We note that, within a pseudopotential formalism, scalar relativistic effects - up to second order in the fine structure constant $\alpha$-may be encapsulated within the expression for the pseudopotential. ${ }^{39}$ Moreover, the largest contribution of the spin-orbit term to the chemical properties has its origin in the core region, and may also be treated within the pseudopotential approximation. We have followed the scheme suggested by Theurich and Hill, ${ }^{40}$ which, coupled to our TDDFT implementation, allows us to discern the effect of spin-orbit in the valence excitations. We obtain in this way, for the atomic ${ }^{2} P_{1 / 2}$ and ${ }^{2} P_{3 / 2}$ spectroscopic lines, 4.85 and $5.30 \mathrm{eV}$ (experimentally they are found at 4.632 and $5.105 \mathrm{eV}) .^{22}$ There is an absolute error of about $0.2 \mathrm{eV}$, but the error in the difference-the spin-orbit splitting-is obtained with the excellent accuracy of $0.025 \mathrm{eV}$.

The absorption cross sections for the set of clusters studied here are shown in Figs. 1-3 for one-dimensional wires, $2 \mathrm{D}$, and 3D structures, respectively. The plots show the results obtained with (black thick line) and without (lighter grey line) the spin-orbit coupling term.

For the wires (Fig. 1), the inclusion of the spin-orbit term has a quite-large effect. For the smallest system, $\mathrm{Au}_{2}$, the splitting of the first peak in the spectrum amounts to almost $1.5 \mathrm{eV}$. The very small peak at low energy is missed experimentally, whereas the following peak (at around $3 \mathrm{eV}$ ) is in good agreement with the main experimental peak at $3.18 \mathrm{eV}^{41,42}$ If spin-orbit coupling is not included, this agreement is slightly worse. ${ }^{43}$ The spin-orbit effect is slightly reduced with increasing number of atoms. However, even for
TABLE I. Polarizabilities per atom (in a.u.) for the 2D and 3D clusters structures studied in this work, calculated either without or with $(+L \cdot S)$ spin-orbit coupling.

\begin{tabular}{ccccccccc}
\hline \hline Cluster size & 1 & 2 & 3 & 4 & 5 & 6 & 7 & 8 \\
\hline$\langle\alpha\rangle(2 \mathrm{D})$ & 5.15 & 5.57 & 5.79 & 5.86 & 6.02 & 5.86 & 6.39 & 6.28 \\
$(+L \cdot S)$ & 5.20 & 5.62 & 6.32 & 5.91 & 6.09 & 5.94 & 6.47 & 6.34 \\
$\langle\alpha\rangle(3 \mathrm{D})$ & & & & 5.95 & 5.96 & 5.62 & 5.59 & 5.48 \\
$(+L \cdot S)$ & & & & 6.03 & 5.89 & 5.62 & 5.63 & 5.46 \\
\hline \hline
\end{tabular}

the largest system studied $\left(\mathrm{Au}_{7}\right)$, spin orbit still changes the relative oscillator strength and the peak positions by as much as $0.5 \mathrm{eV}$. This result is particularly important in the field of electronic transport, where wires of these dimensions often appear in experimental and theoretical setups. As resonances in the conductance arise when hitting an electronic excitation energy, our results indicate that inclusion of spin-orbit coupling is crucial to obtain these resonances with the correct position and intensity. In the case of supported goldchains, ${ }^{35}$ the screening introduced by the substrate hinders this effect, effectively quenching spin orbit leading to more "delocalized $s$-like particle-in-a-box states."

For the case of 2D and 3D structures (Figs. 2 and 3), the effect is still noticeable, but much smaller than for the 1D wires. As the cluster size grows, the density of excited states grows, and the detailed peak structures is washed away by the superposition of spectral widths. Even though there is a clear difference between planar and 3D structures, the latter show fingerprints of the surface plasmon resonance characteristic of gold nanoparticles. For the planar structures, as the system size increases, the difference in the spectra with and without spin orbit is washed out rather soon and after $\mathrm{Au}_{5}$, the spectra are rather similar. In contrast, for 3D structure there are still differences in the peak intensities as well as in several peak splittings. In particular, for the case of $\mathrm{Au}_{20}$, the spin-orbit correction introduces some features in the near UV range which can be detected at experimental resolution (and that are not shown in previous calculations without spin orbit; see Ref. 44 and references therein).

In addition to the absorption spectra (i.e., the imaginary part of the dynamical polarizability), we also report in Table I the static dipole polarizabilities (which we computed with the same numerical technology, making use of the finite field technique) for both the planar and the 3D structures. The atomic value is very similar with and without spin-orbit, in agreement with previous calculations, ${ }^{44}$ and within the error bar of experimental estimates. ${ }^{46}$ For the clusters, the main conclusion that can be drawn is that the effect of the inclusion of spin orbit is also rather small-except maybe in the case of $\mathrm{Au}_{3}$ - and in most cases increasing the polarizability. It is worth noting that the overall relativistic effects in $\mathrm{Au}$ lead to a size contraction, and correspondingly the static polarizability should decrease. The spin-orbit part of the relativistic interactions seems to work in the opposite direction (specially for 2D clusters). The contribution of this effect, however, is much smaller than the scalar relativistic one. The planar structures exhibit, for this cluster size range, even-odd oscillations as reported in previous works. ${ }^{44}$ 
We would like to stress that, by comparing the photoabsorption cross section spectra obtained for different isomers with the same size, it is clear that it carries relevant structural information. Therefore, optical spectroscopy can be used as a noninvasive characterization tool to discern the specific isomer synthesized in experiments and test the predictions for change of shape of supported gold clusters by a static electric field. ${ }^{7}$ We can speculate that the rather strong spin-orbit effects in one-dimensional gold wires could have implications in the development of spintronics devices.

This work was supported in part by the Deutsche Forschungsgemeinschaft (through SFB 658), Spanish MEC (FIS2007-65702-C02-01), Grupos Consolidados UPV/EHU of the Basque Country Government (IT-319-07) European Community e-I3 ETSF project, NANOQUANTA Network of Excellence, CONACyT Mexico (Project No. J-59853-F), and the portuguese FCT (Contract No. SFRH/BD/12712/2003). The computer resources were provided by the Barcelona Supercomputing Center, and the Laboratório de Computação Avançada of the University of Coimbra.

\footnotetext{
${ }^{1}$ The Chemistry of Nanomaterials: Synthesis, Properties and Applications, edited by C. N. R. Rao, A. Müller, and A. K. Cheetham (Wiley, New York, 2004); J. A. Alonso, Structure and Properties of Atomic Clusters (Imperial College Press, London, 2005); See also the dedicated section about clusters on Science 271, 920 (1996); For gold clusters, see, e.g., P. Schwerdtfeger, Angew. Chem., Int. Ed. 42, 1892 (2003).

${ }^{2}$ U. Heiz and U. Landman, Nanocatalysis (Springer, New York, 2006).

${ }^{3}$ S. Palomba, L. Novotny, and R. E. Palmera, Opt. Commun. 281, 480 (2008); U. Kreibig and M. Vollmer, Optical Properties of Metal Clusters (Springer, Berlin, 1995); J. Zheng, C. Zhang, and R. M. Dickson, Phys. Rev. Lett. 93, 077402 (2004)

${ }^{4}$ J. Guzman and B. C. Gates, Nano Lett. 1, 689 (2001).

${ }^{5}$ A. Sanchez, S. Abbet, U. Heiz, W.-D. Schneider, H. Häkkinen, R. N. Barnett, and U. Landman, J. Phys. Chem. A 103, 9573 (1999).

${ }^{6}$ S. Liu, R. Maoz, G. Schmid, and J. Sagiv, Nano Lett. 2, 1055 (2002).

${ }^{7}$ B. Yoon and U. Landman, Phys. Rev. Lett. 100, 056102 (2008)

${ }^{8}$ N. Toshima, T. Yonezawa, and K. Kushihashi, J. Chem. Soc., Faraday Trans. 89, 2537 (1993).

${ }^{9}$ W. A. de Heer, Rev. Mod. Phys. 65, 611 (1993); M. Brack, ibid. 65, 677 (1993).

${ }^{10}$ J. Tiggesbäumker, L. Köller, and K. H. Meiwes-Broer, Chem. Phys. Lett. 260, 428 (1996)

${ }^{11}$ J. Tiggesbäumker, L. Köller, K.-H. Meiwes-Broer, and A. Liebsch, Phys. Rev. A 48, R1749 (1993).

${ }^{12}$ B. Palpant, B. Prével, J. Lermé, E. Cottancin, M. Pellarin, M. Treilleux, A. Perez, J. L. Vialle, and M. Broyer, Phys. Rev. B 57, 1963 (1998).

${ }^{13}$ Ll. Serra and A. Rubio, Phys. Rev. Lett. 78, 1428 (1997); K. Yabana and G. F. Bertsch, Phys. Rev. A 60, 3809 (1998)

${ }^{14}$ P. Pyykko, Angew. Chem., Int. Ed. 43, 4412 (2004).

${ }^{15}$ F. Furche, R. Ahlrichs, P. Weis, C. Jacob, S. Gilb, T. Bierweiler, and M. M. Kappes, J. Chem. Phys. 117, 6982 (2002); S. Gilb, P. Weis, F. Furche, R. Ahlrichs, and M. M. Kappes, ibid. 116, 4094 (2002).

${ }^{16}$ E. M. Fernández, J. M. Soler, and L. C. Balbás, Phys. Rev. B 73, 235433 (2006).

${ }^{17}$ H. Häkkinen, B. Yoon, U. Landman, X. Li, H.-J. Zhai, and L.-S. Wang, J. Phys. Chem. A 107, 6168 (2003).

${ }^{18}$ H. Häkkinen, M. Moseler, and U. Landman, Phys. Rev. Lett. 89, 033401
}

(2002).

${ }^{19}$ Some work has been reported for other properties, i.e., magnetic anisotropies: L. Fernández-Seivane and J. Ferrer, Phys. Rev. Lett. 99, 183401 (2007).

${ }^{20}$ E. Eliav and U. Kaldor, Phys. Rev. A 49, 1724 (1994); I. Itkin and A. Zaitsevskii, Chem. Phys. Lett. 374, 143 (2003); X. Wang, X. Wan, H. Zhou, S. Takami, M. Kubo, and A. Miyamoto, J. Mol. Struct.: THEOCHEM 579, 221 (2002).

${ }^{21}$ Up to now, all TDDFT calculations of the static polarizabilities and the absorption spectra of $\mathrm{Au}_{n}$ clusters $(n=2-14,20)$ (see, for example, Refs. 44 and 45 , and references therein) have neglected the impact of relativity beyond its effect on the geometrical structure.

${ }^{22}$ C. E. Moore, Atomic Energy Levels (Natl. Bur. Stand. U.S. GPO, Wahingnton, DC, 1948)

${ }^{23}$ Time-Dependent Density-Functional Theory, edited by M. A. L. Marques, C. A. Ullrich, F. Nogueira, A. Rubio, K. Burke, and E. K. U. Gross, Lecture Notes in Physics Vol. 706 (Springer Verlag, Berlin, 2006).

${ }^{24}$ K. Yabana and G. F. Bertsch, Phys. Rev. B 54, 4484 (1996); K. Yabana and G. F. Bertsch, Phys. Rev. A 58, 2604 (1999).

${ }^{25}$ M. A. L. Marques, A. Castro, G. F. Bertsch, and A. Rubio, Comput. Phys. Commun. 151, 60 (2003); A. Castro, M. A. L. Marques, H. Appel, M. Oliveira, C. Rozzi, X. Andrade, F. Lorenzen, E. K. U. Gross, and A. Rubio, Phys. Status Solidi B 243, 2465 (2006); M. A. L. Marques, A. Castro, and A. Rubio, J. Chem. Phys. 115, 3006 (2001).

${ }^{26}$ L. Xiao and L. Wang, Chem. Phys. Lett. 392, 452 (2004).

${ }^{27}$ R. M. Olson, S. Varganov, M. S. Gordon, H. Metiu, S. Chretien, P. Piecuch, K. Kowalski, S. A. Kucharski, and M. Musial, J. Am. Chem. Soc. 127, 1049 (2005)

${ }^{28}$ R. Car and M. Parrinello, Phys. Rev. Lett. 55, 2471 (1985); We used the CPMD code, version 3.5, developed by J. Hutter et al., at MPI für Festkörperforschung and IBM Research Laboratory (1990-2001).

${ }^{29}$ N. Troullier and J. Martins, Phys. Rev. B 43, 1993 (1991).

${ }^{30}$ H. Grönbeck and W. Andreoni, Chem. Phys. 262, 1 (2000).

${ }^{31}$ J. Rogan, R. Ramírez, A. H. Romero, and M. Kiwi, Eur. Phys. J. D 28 , 219 (2004).

${ }^{32}$ Atomic gold chains can be created by stretching gold nanocontacts, or by deposition on stepped surfaces (Ref. 35). Their interatomic distance can vary substantially; see, e.g., N. V. Skorodumova and S. I. Simak, Phys. Rev. B 67, 121404(R) (2003).

${ }^{33}$ M. D. Morse, Chem. Rev. (Washington, D.C.) 86, 1049 (1986)

${ }^{34}$ U. Landman, W. D. Luedtke, N. A. Burnham, and R. J. Colton, Science 248, 454 (1990); U. Landman and W. D. Luedtke, J. Vac. Sci. Technol. B 9, 414 (1991); H. Ohnishi, Y. Kondo, and K. Takayanagi, Nature (London) 395, 780 (1998); H. Häkkinen, R. N. Barnett, A. G. Scherbakov, and U. Landman, J. Phys. Chem. B 104, 9063 (2000).

${ }^{35}$ N. Nilius, T. M. Wallis, and W. Ho, Science 297, 1853 (2002).

${ }^{36}$ In contrast to structural properties, the optical spectra of small metal and semiconductor clusters in the visible range turn out to be, for fixed ionic configuration, not much sensitive to the use of the LDA or PBE-GGA for exchange and correlation effects. See, for example, M. A. L. Marques, A. Castro, and A. Rubio, J. Chem. Phys. 115, 3006 (2001).

${ }^{37}$ S. Engel, A. Höck, and S. Varga, Phys. Rev. B 63, 125121 (2001)

${ }^{38}$ M. J. T. Oliveira and F. Nogueira, Comput. Phys. Commun. 178, 524 (2008).

${ }^{39}$ G. B. Bachelet, D. R. Hamann, and M. Schlüter, Phys. Rev. B 26, 4199 (1982).

${ }^{40}$ G. Theurich and N. A. Hill, Phys. Rev. B 64, 073106 (2001).

${ }^{41}$ L. L. Ames and R. F. Barrow, Trans. Faraday Soc. 63, 39 (1967).

${ }^{42}$ G. A. Bishea and M. D. Morse, J. Chem. Phys. 95, 5646 (1991).

${ }^{43} \mathrm{We}$ have also performed calculations for $\mathrm{Au}_{2}$ at the bulk interatomic distance $(2.9 \AA)$. The whole absorption spectrum is redshifted, as expected, and the shapes do not change significantly in the optical region; in particular the spin-orbit splitting stays roughly the same. 